\title{
On Particle Production in Lead-Gold Collision and Azimuthal Anisotropy at Top SPS Energy
}

\author{
Bao-Chun Li and Zhao Zhang \\ Department of Physics, Shanxi University, Taiyuan, Shanxi 030006, China \\ Correspondence should be addressed to Bao-Chun Li; libc2010@163.com
}

Received 25 March 2014; Accepted 26 April 2014; Published 18 May 2014

Academic Editor: Chen Wu

Copyright ( 2014 B.-C. Li and Z. Zhang. This is an open access article distributed under the Creative Commons Attribution License, which permits unrestricted use, distribution, and reproduction in any medium, provided the original work is properly cited. The publication of this article was funded by SCOAP S $^{3}$

In a multisource thermal model, we analyze the dependence of elliptic flow $v_{2}$ on the transverse momentum $P_{T}$. The model results are compared with the data of $\pi^{-}, K_{S}^{0}, p$, and $\Lambda$ measured in $\mathrm{Pb}+\mathrm{Au}$ collisions at top SPS energy, $17.3 \mathrm{GeV}$. It is found that the azimuthal anisotropy in the evolution process of high-energy collisions is correlated highly to the number of participant nucleons.

\section{Introduction}

Collective flows are a common phenomenon of heavy ion collisions at high energy [1-5]. In a noncentral collision of two spherical nuclei, the overlapping zone between the two nuclei is not circular but it rather takes an almond shape, where pressure gradients are formed due to the azimuthal anisotropy. Then, the initial spatial anisotropy of the overlapping zone is converted into momentum space anisotropy of the particle distribution. The momentum distribution of emitted particles is quantified in terms of the Fourier expansion for the particle invariant azimuthal distribution [6]. These are defined by

$$
E \frac{d N}{d^{3} p}=\frac{d N}{2 \pi p_{T} d p_{T} d y}\left(1+2 \sum_{n=1}^{\infty} v_{n} \cos \left[n\left(\phi-\Psi_{n}\right)\right]\right),
$$

where $\phi_{n}$ and $\Psi_{n}$ represent the azimuthal angle of an emitted particle in laboratory reference frame and the azimuthal angle of the reaction plane in the same frame, respectively. The $v_{n}$ represents the magnitude of the $n$ th-order anisotropy. The second-order coefficient of the anisotropy flow $v_{2}$ is called the elliptic flow, which is related to the almond shape mentioned above. The elliptic flow is one of the key observables measured at the RHIC, where large values of $v_{2}$ are considered as a sign of collectivity in the system created in the high-energy collisions. Recently, an event-by-event ideal hydrodynamic model is used to study the elliptic flow of thermal photons in $\mathrm{Au}+\mathrm{Au}$ collisions at RHIC and $\mathrm{Pb}+\mathrm{Pb}$ collisions at the LHC, and the results show that there is a persistent tension between experimental data and photons of hydrodynamics [7].

In this paper, we will discuss the elliptic flow in $\mathrm{Pb}+\mathrm{Au}$ collision at $\sqrt{s_{\mathrm{NN}}}=17.3 \mathrm{GeV}$, which is top SPS energy. To extract properties of the matter created in the asymmetry reaction, we improve a multisource thermal model, which was used to describe the particle production in our previous work [8].

\section{Azimuthal Anisotropy and Number of Participant Nucleons}

The initial transverse coordinate-space anisotropy of the participant region is converted into an azimuthal momentumspace anisotropy [9]. But we obtain the evolution information of such matter by final-state particles measured in detectors [10]. The transverse momentum $p_{T}^{\prime}$ of the particles has an invariant form in different reference fames:

$$
f_{p_{T}^{\prime}}\left(p_{T}^{\prime}\right)=\frac{1}{\sigma^{2}} \exp \left[-\frac{p_{T}^{\prime 2}}{2 \sigma^{2}}\right],
$$

which is called Rayleigh distribution.

When two ultrarelativistic nuclei collide at a nonzero impact parameter, their overlap area in the transverse plane has a short axis which is parallel to the impact parameter and a long axis which is perpendicular to the impact parameter. 


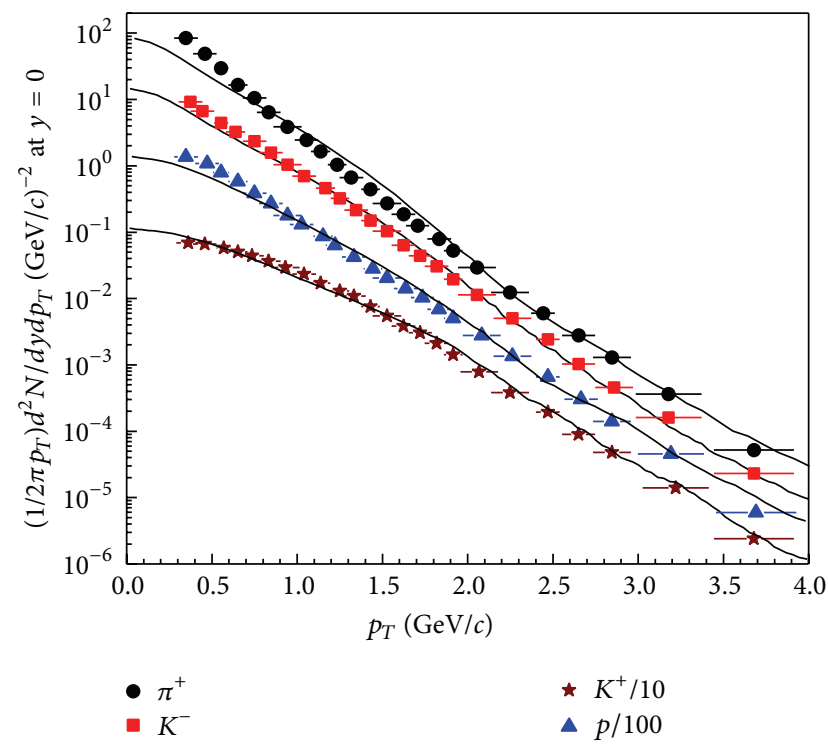

Figure 1: Invariant $p_{T}$-differential yields of $\pi^{+}, K^{+}, K$, and protons. The experimental data [16] are denoted by the scattered symbols. The theoretical results are denoted by the solid line.

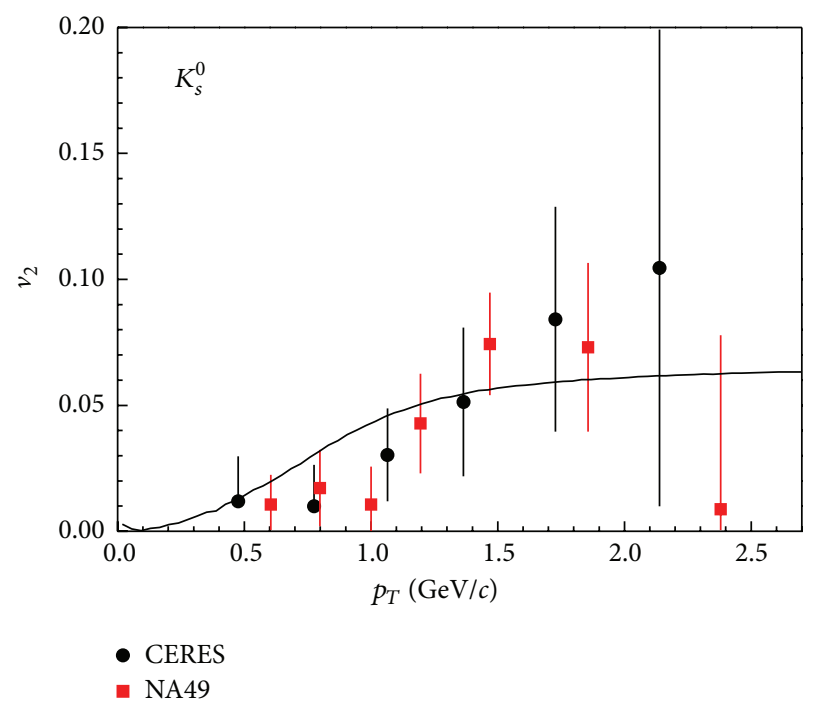

Figure 2: Differential elliptic flow $v_{2}\left(p_{T}\right)$ of $K_{S}^{0}$. The CERES data [17] and the NA49 data [18] are denoted by the scattered symbols. The theoretical results are denoted by the solid line.

Considering the mechanics effect, these sources depart from the isotropic emission. The distribution functions of $p_{x}^{*}$ and $p_{y}^{*}$ are

$$
\begin{aligned}
& p_{x}^{*}=A_{x} p_{x}^{\prime}+B_{x}, \\
& p_{y}^{*}=A_{y} p_{y}^{\prime}+B_{y},
\end{aligned}
$$

where $A_{x, y}$ and $B_{x, y}$ denote the expansion coefficient of the source and the movement coefficient of the source, respectively. For Maxwell's ideal gas, $A_{x, y}=1$ and $B_{x, y}=$ 0 , and the nonexpanding source has no contribution to the

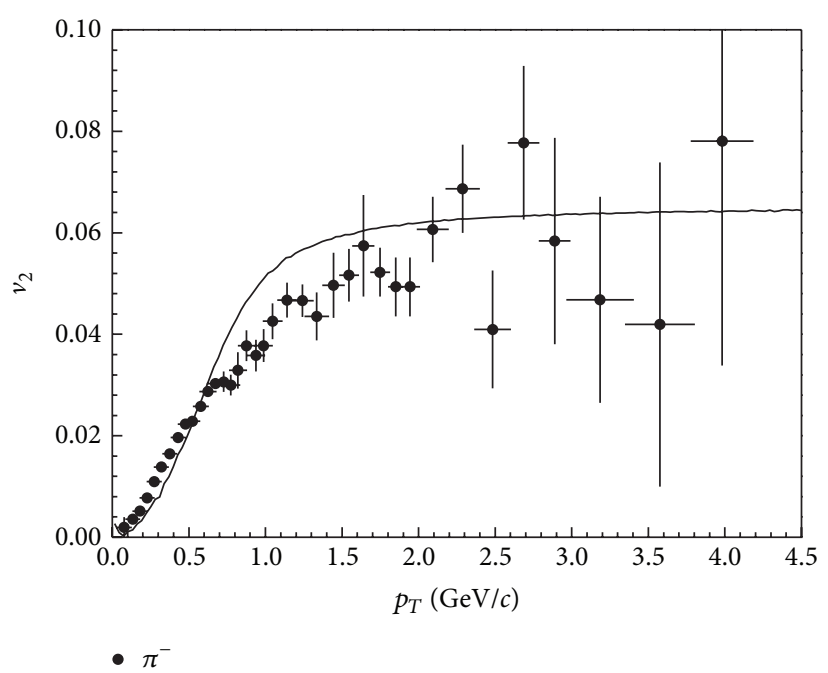

FIGURE 3: Differential elliptic flow $v_{2}\left(p_{T}\right)$ of negative pion candidates after the HBT correction. The CERES data [17] are denoted by the scattered symbols. The theoretical results are denoted by the solid line.

collective flow. The particle distribution is $f_{p_{T}^{*}, \phi^{*}}\left(p_{T}^{*}, \phi^{*}\right)$. In the final state, the normalized distribution of total particles can be expressed as

$$
f_{p_{T}, \phi}\left(p_{T}, \phi\right)=u f_{p_{T}^{\prime}}\left(p_{T}^{\prime}\right)+(1-u) f_{p_{T}^{*}, \phi}\left(p_{T}^{*}, \phi\right),
$$

where $u$ and $1-u$ denote the contribution of isotropic sources and anisotropic sources, respectively. The $1-u$ is proportional to the number of nucleons participating $N_{\text {part }}$ :

$$
u=1-k \frac{N_{\text {part }}}{A_{\mathrm{Pb}}+A_{\mathrm{Au}}},
$$

where $A_{\mathrm{Pb}}$ and $A_{\mathrm{Au}}$ are the mass numbers of the $\mathrm{Pb}$ and $\mathrm{Au}$, respectively.

In the Monte Carlo calculation, the transverse momenta are given by

$$
\begin{aligned}
p_{T}^{*}=( & {\left[A_{x} \sigma \sqrt{-2 \ln r_{1}} \cos \left(2 \pi r_{2}\right)+B_{x}\right]^{2} } \\
& \left.+\left[A_{y} \sigma \sqrt{-2 \ln r_{3}} \cos \left(2 \pi r_{4}\right)+B_{y}\right]^{2}\right)^{1 / 2},
\end{aligned}
$$

where $r_{1}, r_{2}, r_{3}$, and $r_{4}$ denote random numbers in $[0,1]$. According to the measurement in experiments, the elliptic flow in the model is defined as

$$
v_{2}=\left\langle\frac{p_{x}^{2}-p_{y}^{2}}{p_{x}^{2}+p_{y}^{2}}\right\rangle .
$$

It is interesting to note that several different definitions of centralities in $A+A$ collision were given through other quantities because they are not a directly measurable quantity [11]. The centrality can usually be calculated by multiplicity distribution or fractional cross-section or impact parameter 

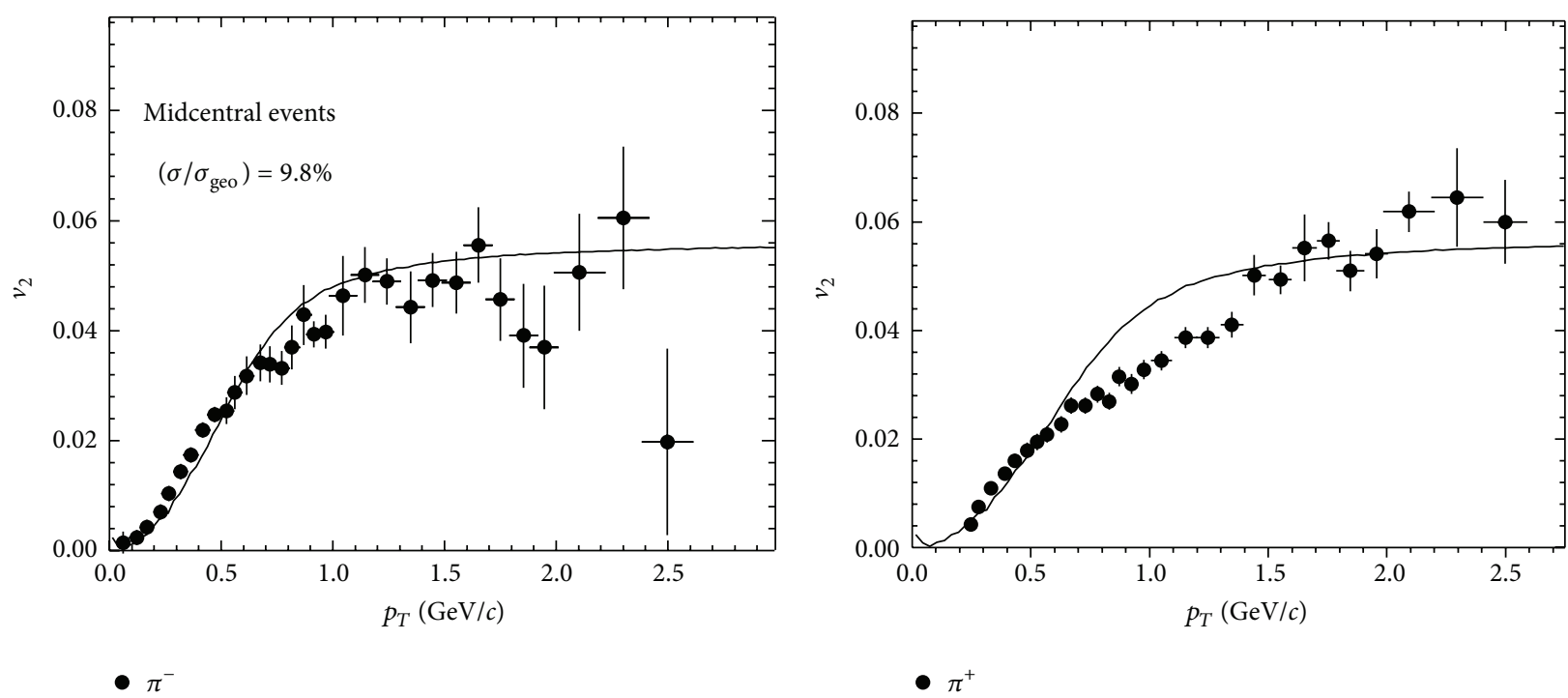

Figure 4: The same as in Figure 4, but for $\pi^{-}$and $\pi^{+}$candidates.

$b, b^{2} /\left(R_{\mathrm{Pb}}+R_{\mathrm{Au}}\right)^{2}$ [12]. The $R_{\mathrm{Pb}}$ and $R_{\mathrm{Au}}$ are radii of $\mathrm{Pb}$ and $\mathrm{Au}$, respectively. Theoretically, the number of participant nucleons in a collision of $\mathrm{Pb}+\mathrm{Au}$ at the impact parameter $b$ is

$$
N_{\text {part }}(b)=N_{\text {part }}^{\mathrm{Pb}}(b)+N_{\text {part }}^{\mathrm{Au}}(b) .
$$

They can be estimated easily in the geometry method [13]:

$$
\begin{aligned}
& N_{\mathrm{part}}^{\mathrm{Pb}}(b) \\
&=\int d V \rho_{\mathrm{Pb}}\left(\sqrt{x^{2}+(b-y)^{2}+z^{2}}\right) \\
& \quad \times \theta\left[R_{\mathrm{Pb}}-\sqrt{x^{2}+(b-y)^{2}+z^{2}}\right] \theta\left[R_{\mathrm{Au}}-\sqrt{x^{2}+y^{2}}\right], \\
& N_{\mathrm{part}}^{\mathrm{Au}}(b) \\
&=\int d V \rho_{\mathrm{Au}}\left(\sqrt{x^{2}+y^{2}+z^{2}}\right) \\
& \quad \times \theta\left[R_{\mathrm{Au}}-\sqrt{x^{2}+y^{2}+z^{2}}\right] \theta\left[R_{\mathrm{Pb}}-\sqrt{x^{2}+(b-y)^{2}}\right] .
\end{aligned}
$$

The nuclear density function $\rho_{\mathrm{Pb} / \mathrm{Au}}$ is the Woods-Saxon density profile [14]:

$$
\rho_{\mathrm{Pb} / \mathrm{Au}}(r)=\frac{\rho_{0}}{1+\exp \left[\left(r-R_{\mathrm{Pb} / \mathrm{Au}}\right) / c\right]},
$$

where $\rho_{0}$ refers to normal nuclear density and $c \approx 0.54 \mathrm{fm}$ stands for the nuclear diffusion edge from low energy electron-nucleus scattering experiments [15].

First, in order to check the reliability of the model, Figure 1 presents the transverse momentum spectra of charged pions, charged kaons and protons at midrapidity for $\mathrm{Pb}-\mathrm{Pb}$ collisions at $\sqrt{s_{\mathrm{NN}}}=17.3 \mathrm{GeV}$. The symbols denote

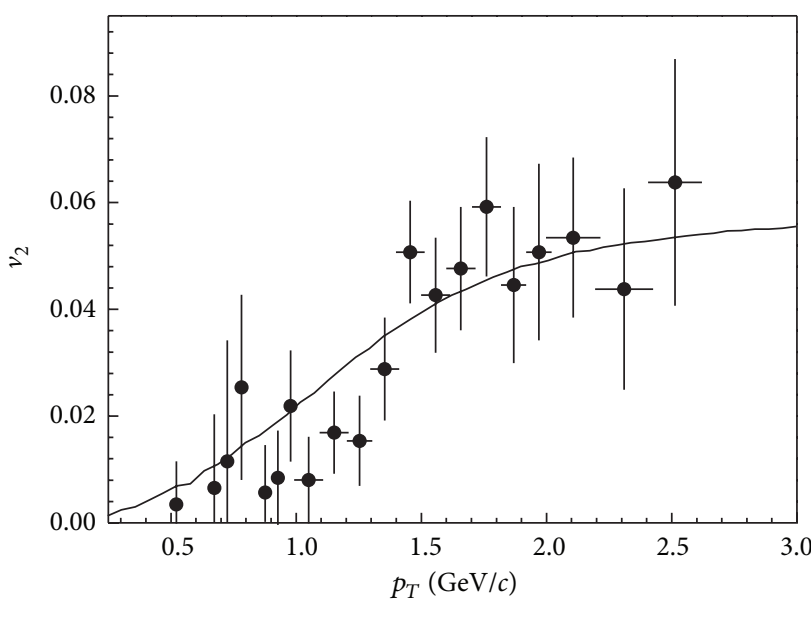

- $p$

FIgURE 5: The same as in Figure 4, but for the proton.

the data measured by NA49 Collaboration [16]. The curves denote our results with $A_{x}=3.10 \pm 0.45$ and $\sigma=0.44 \pm 0.14$. The calculated results agree with the experimental data except for the region of $p_{T}<0.6 \mathrm{GeV} / c$.

Figure 2 shows elliptic flows $v_{2}$ of $K_{S}^{0}$ produced in $\mathrm{Pb}$ Au collisions at $\sqrt{s_{\mathrm{NN}}}=17.3 \mathrm{GeV}$. The symbols are the data measured by the CERES Collaboration [17] and NA49 Collaboration [18]. Collision centralities are $5.3-13 \%$ of $\sigma_{\text {geo }}$ for CERES and top 13\% for NA49 data. The curves are our results with $A_{x}=3.10 \pm 0.45$, which depends on collision energies and the centrality. The other parameters used in the calculations are $k=0.24 \pm 0.04$ and $\sigma=0.45 \pm$ 0.18 . The observed $v_{2}$ increases with increasing $p_{T}$ at the low transverse momentum and saturates in $p_{T}>2 \mathrm{GeV} / c$. The modeling results are in approximate agreement with the experimental data. Figures 3 and 4 show elliptic flows 


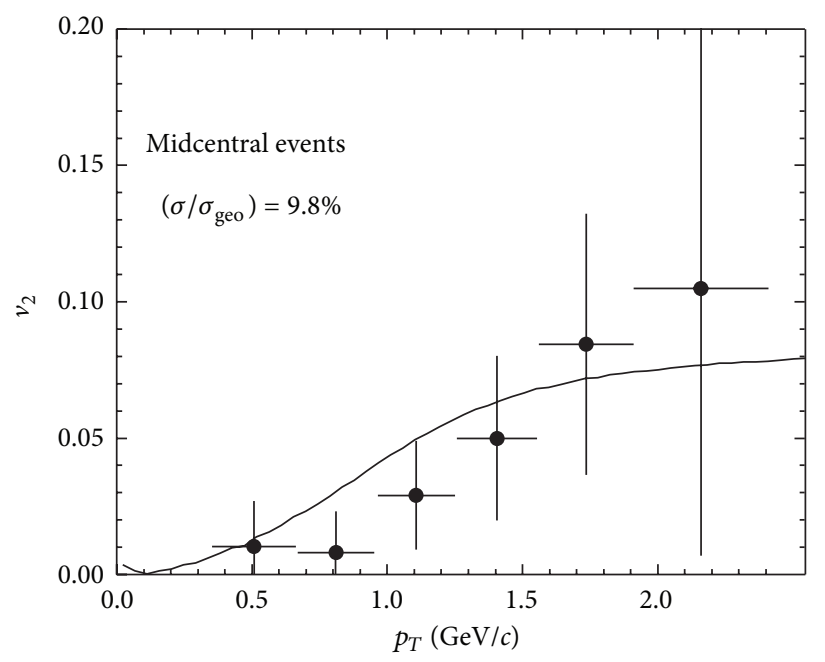

- $K_{s}^{0}$

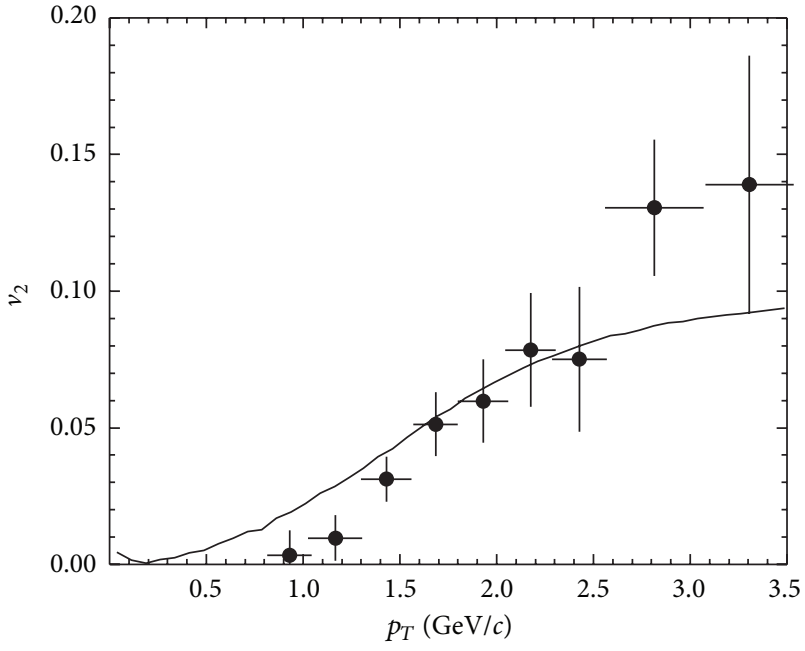

- $\Lambda$

Figure 6: The same as in Figure 4, but for $K_{S}^{0}$ mesons and $\Lambda$ hyperons.
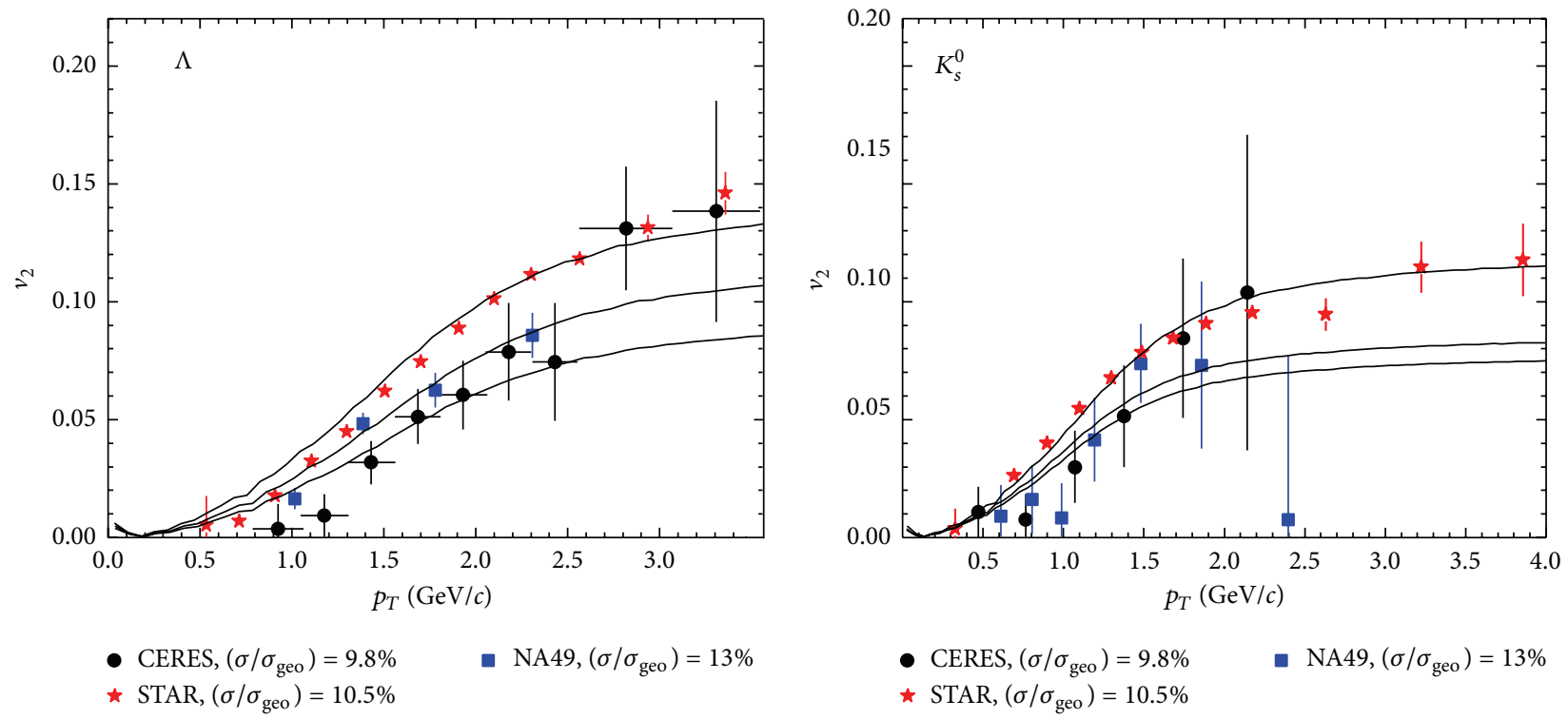

FIgURE 7: Comparison of $\Lambda$ and $K_{S}^{0}$ elliptic flow between CERES and NA49 [19] and STAR [20].

$v_{2}$ of $p_{T}$ for $\pi^{-}$and $\pi^{+}$produced in $\mathrm{Pb}-\mathrm{Au}$ collisions at $\sqrt{s_{\mathrm{NN}}}=17.3 \mathrm{GeV}$. The symbols denote the data measured by the CERES Collaboration [17]. The curves denote our results. One sees again that the model can approximately describe the elliptic flows in $\mathrm{Pb}-\mathrm{Au}$ collisions at the top SPS energy.

In Figure 5, we show differential elliptic flows $v_{2}\left(p_{T}\right)$ of protons in $\mathrm{Pb}-\mathrm{Au}$ collisions at $\sqrt{s_{\mathrm{NN}}}=17.3 \mathrm{GeV}$. In the given centrality, the dependence of $v_{2}$ on $p_{T}$ is similar to that for the pion. In Figure 6, we present differential elliptic flows $v_{2}\left(p_{T}\right)$ of $K_{S}^{0}$ mesons and $\Lambda$ hyperons in $\mathrm{Pb}-\mathrm{Au}$ collisions at $\sqrt{s_{\mathrm{NN}}}=17.3 \mathrm{GeV}$. We also see the same behavior for the same centrality like the above figures. In order to understand the azimuthal anisotropy of final-state particles in the asymmetric collision, Figure 7 gives a comparison to the results of NA49 [19] at the same energy $\sqrt{s_{\mathrm{NN}}}=17.3 \mathrm{GeV}$ and gives a comparison to STAR results [20] at $\sqrt{s_{\mathrm{NN}}}=$ $200 \mathrm{GeV}$. The NA49 and CERES data are in reasonably good agreement. In order to compare STAR to CERES results, the former have been rescaled to the centrality used in the CERES experiment. The $v_{2}$ values measured at the RHIC energy are 15-20\% higher due to the higher beam energy. Our results are in approximate agreement with the data measured by the three collaborations.

\section{Conclusions}

In the above discussions, we have investigated the azimuthal anisotropy by using the elliptic flow $v_{2}$ versus the transverse 
momentum $p_{T}$ in $\mathrm{Pb}+\mathrm{Au}$ collisions at the top SPS energy $\sqrt{s_{\mathrm{NN}}}=17.3 \mathrm{GeV}$. The amplitude of the azimuthal anisotropy can be measured by the elliptic flow, which is a sensitive probe of the matter created in the collisions. We improve the multisource statistical model to discuss the transverse momentum dependence of elliptic flows for the particles produced in the asymmetric system, $\mathrm{Pb}-\mathrm{Au}$ collision at the top SPS energy. Considering the collision geometry, the modelling results are obtained in the framework of the multisource statistical model. The results agree approximately with the data of the CERN-CERES/NA45 Collaboration. More importantly, this model has partly described the particle production in highenergy collisions [8]. In the descriptions of elliptic flows $v_{2}\left(p_{T}\right)$ in the asymmetric system $(\mathrm{Pb}+\mathrm{Au}$ collision $)$ at SPS energies, the present work is a new successful attempt.

\section{Conflict of Interests}

The authors declare that there is no conflict of interests regarding the publication of this paper.

\section{Acknowledgments}

This work is supported by the National Natural Science Foundation of China under Grants no. 11247250, no. 11005071, and no. 10975095, the National Fundamental Fund of Personnel Training under Grant no. J1103210, and the Shanxi Provincial Natural Science Foundation under Grants no. 2013021006 and no. 2011011001.

\section{References}

[1] B. Abelev, J. Adam, D. Adamová et al., " $D$ Meson eliptic flow in noncentral $\mathrm{Pb}-\mathrm{Pb}$ collisions at $\sqrt{s_{N N}}=2.76 \mathrm{TeV}$," Physical Review Letters, vol. 111, Article ID 102301, 2013.

[2] E. Retinskaya, M. Luzum, and J.-Y. Ollitrault, "Constraining models of initial conditions with elliptic and triangular flow data," Physical Review C, vol. 89, Article ID 014902, 2014.

[3] J. Xu, T. Song, C. M. Ko, and F. Li, "Elliptic flow splitting as a probe of the QCD phase structure at finite baryon chemical potential," Physical Review Letters, vol. 112, Article ID 012301, 2014.

[4] B.-C. Li and L.-L. Wang, "Entropy in scalar O(n) model with a spontaneously broken symmetry," International Journal of Modern Physics A, vol. 24, no. 30, pp. 5725-5736, 2009.

[5] B.-C. Li and M. Huang, "Strongly coupled matter near phase transition," Journal of Physics G, vol. 36, Article ID 064062, 2009.

[6] C. Shen, U. W. Heinz, J.-F. Paquet, I. Kozlov, and C. Gale, "Anisotropic flow of thermal photons as a quark-gluon plasma viscometer," http://arxiv.org/abs/1308.2111. In Press.

[7] R. Chatterjee, H. Holopainen, I. Helenius, T. Renk, and K. J. Eskola, "Elliptic flow of thermal photons from an event-byevent hydrodynamic model," Physical Review C, vol. 88, Article ID 034901, 2013.

[8] L. Bao-Chun, W. Ya-Zhou, L. Fu-Hu, W. Xin-Jian, and D. YouEr, "Particle production in relativistic $p p(\bar{p})$ and $A A$ collisions at RHIC and LHC energies with Tsallis statistics using the twocylindrical multisource thermal model," Physical Review D, vol. 89, Article ID 054014, 2014.
[9] B.-C. Li, Y.-Y. Fu, L.-L. Wang, and F.-H. Liu, "Dependence of elliptic flows on transverse momentum and number of participants in $\mathrm{Au}+\mathrm{Au}$ collisions at $\sqrt{s_{N N}}=200 \mathrm{GeV}$,' Journal of Physics G, vol. 40, Article ID 025104, 2013.

[10] R. A. Soltz, I. Garishvili, M. Cheng et al., "Constraining the initial temperature and shear viscosity in a hybrid hydrodynamic model of $\sqrt{s_{N N}}=200 \mathrm{GeV} \mathrm{Au}+$ Au collisions using pion spectra, elliptic flow, and femtoscopic radii," Physical Review C, vol. 87, Article ID 044901, 2013.

[11] B.-C. Li, Y. Fu, E.-Q. Wang, L.-L. Wang, and F.-H. Liu, "Transverse momentum dependence of charged and strange hadron elliptic flows in $\mathrm{Cu}-\mathrm{Cu}$ collisions," Journal of Physics $\mathrm{G}$, vol. 39, no. 8, Article ID 085109, 2012.

[12] W. Broniowski and W. Florkowski, "Geometric relation between centrality and the impact parameter in relativistic heavy-ion collisions," Physical Review C, vol. 65, Article ID 024905, 2002.

[13] B.-C. Li, Y.-Y. Fu, L.-L. Wang, and F.-H. Liu, "Transverse momentum, centrality, and participant nucleon number dependence of elliptic flow," Advances in High Energy Physics, vol. 2013, Article ID 908046, 7 pages, 2013.

[14] M. L. Miller, K. Reygers, S. J. Sanders, and P. Steinberg, "Glauber modeling in high-energy nuclear collisions," Annual Review of Nuclear and Particle Science, vol. 57, pp. 205-243, 2007.

[15] H. de Vries, C. W. de Jager, and C. de Vries, "Nuclear chargedensity-distribution parameters from elastic electron scattering," Atomic Data and Nuclear Data Tables, vol. 36, no. 3, pp. 495-536, 1987.

[16] C. Alt, T. Anticic, B. Baatar et al., "High transverse momentum hadron spectra at $\sqrt{s_{N N}}=17.3 \mathrm{GeV}$ in $\mathrm{Pb}+\mathrm{Pb}$ and $p+p$ collisions," Physical Review C, vol. 77, Article ID 034906, 2008.

[17] D. Adamova, G. Agakichievb, A. Andronicet et al., "Elliptic flow of charged pions, protons and strange particles emitted in $\mathrm{Pb}+\mathrm{Au}$ collisions at top SPS energy," Nuclear Physics A, vol. 894, pp. 41-73, 2012.

[18] C. Blume, "Recent results from the NA49 experiment," Journal of Physics G, vol. 35, Article ID 044004, 2008.

[19] G. Stefanek, C. Alt, T. Anticic et al., "Anisotropic flow of strange particles at SPS," in Proceedings of the 3rd Edition of the Conference Critical Point and the Onset of Deconfinement (CPOD '06), p. 30, PoS, Florence, Italy, July 2006.

[20] M. Oldenburg, "Centrality dependence of azimuthal anisotropy of strange hadrons in $200 \mathrm{GeV} \mathrm{Au}+\mathrm{Au}$ collisions," Journal of Physics G, vol. 32, article S563, 2006. 

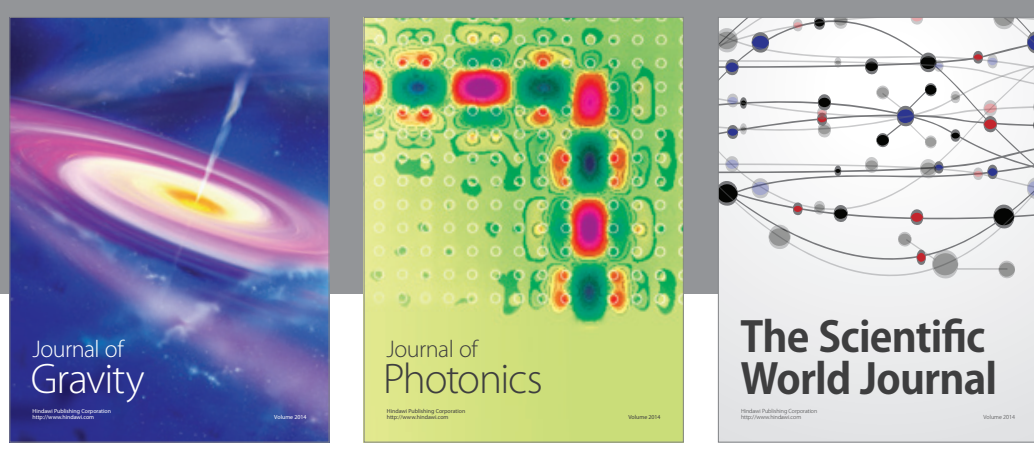

The Scientific World Journal
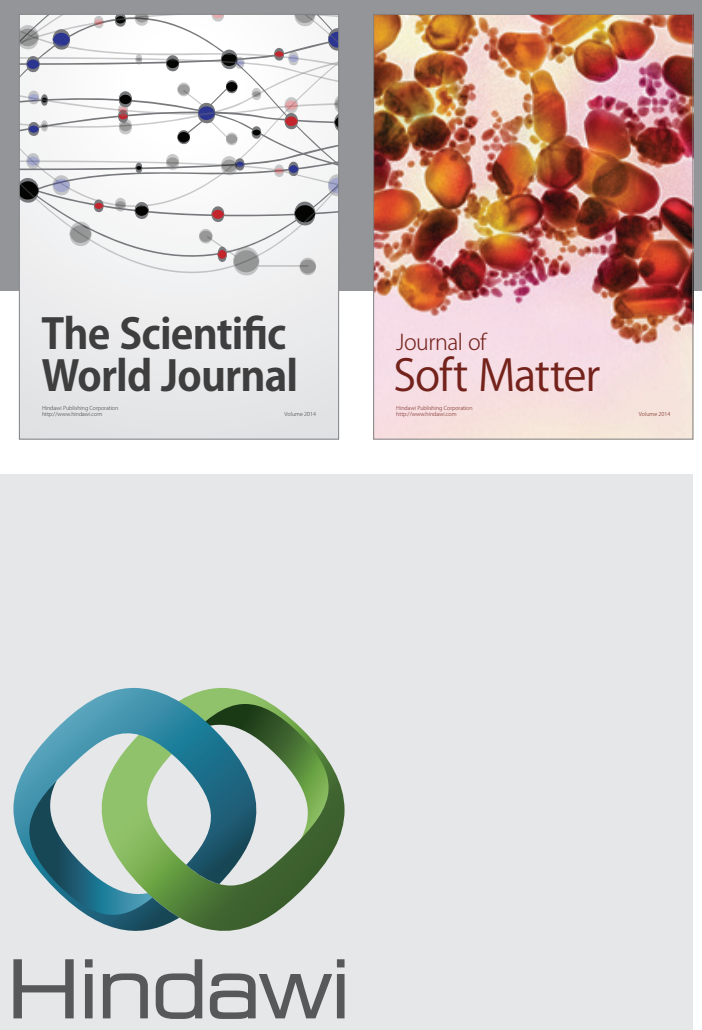

Submit your manuscripts at

http://www.hindawi.com

nternational Journal of

Statistical Mechanics
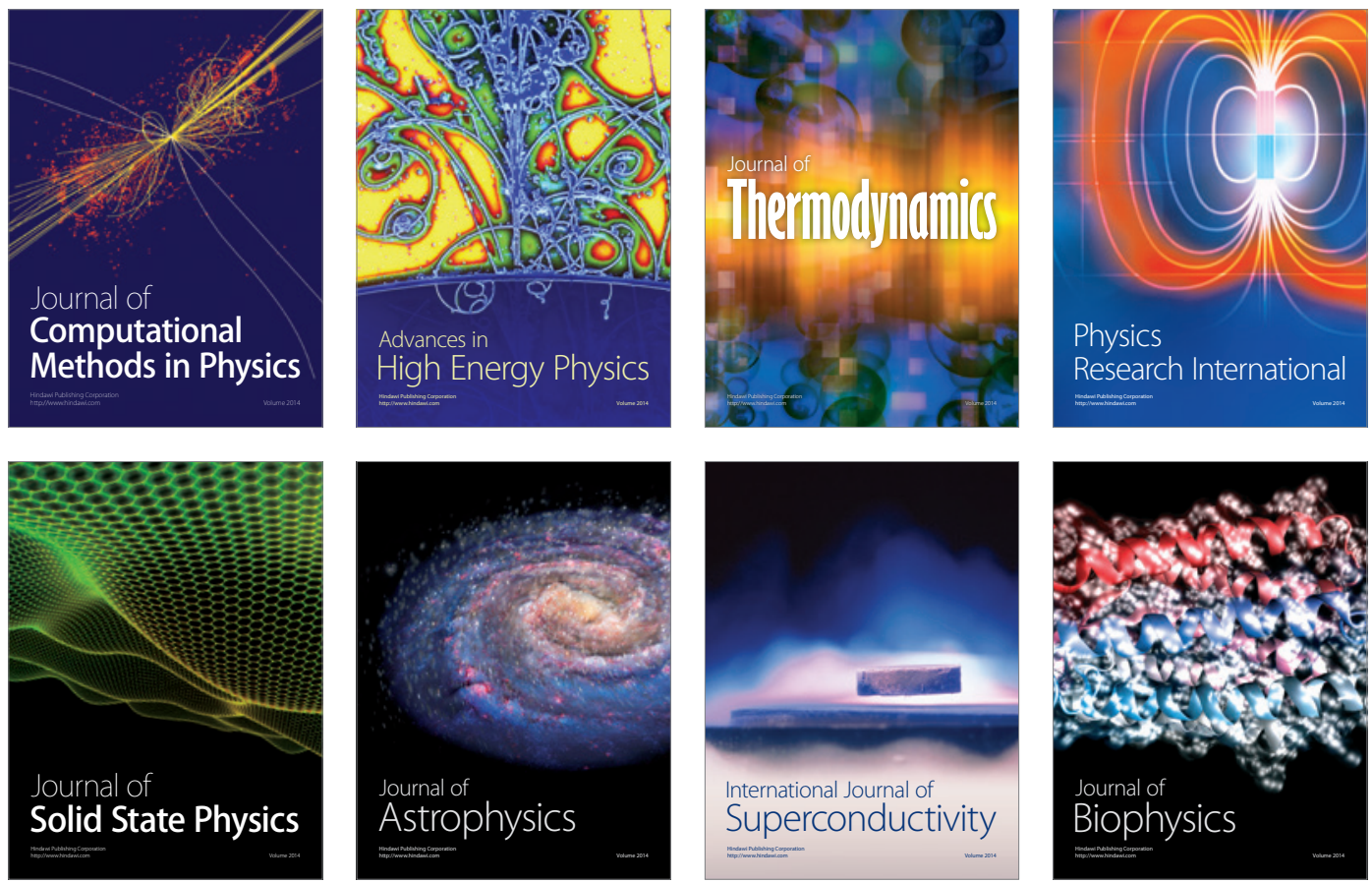
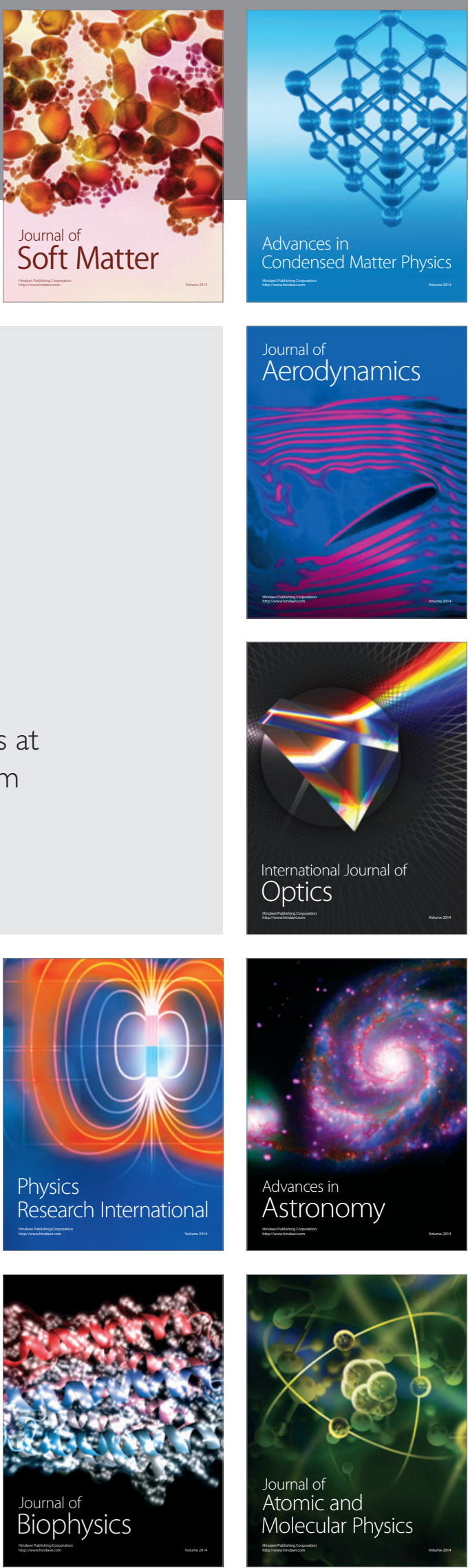